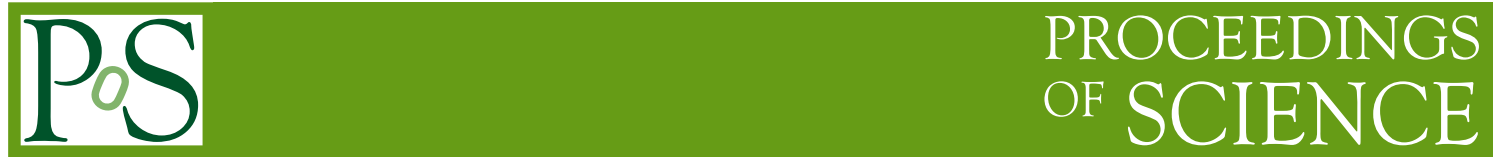

\title{
Hard (State) Problems
}

\author{
John A. Tomsick* \\ Space Science Laboratory, 7 Gauss Way, University of California, Berkeley, CA 94720-7450, \\ USA \\ E-mail: jtomsick@ssl.berkeley.edu
}

For microquasars, the one time when these systems exhibit steady and powerful jets is when they are in the hard state. Thus, our understanding of this state is key to learning about the disk/jet connection. Recent observational and theoretical results have led to questions about whether we really understand the physical properties of this state, and even our basic picture of this state is uncertain. Here, I discuss some of the recent developments and possible problems with our understanding of this state. Overall, it appears that the strongest challenge to the standard truncated disk picture is the detection of broad iron features in the X-ray spectra, and it seems that either there is a problem with the truncated disk picture or there is a problem with the relativistic reflection models used to explain the broad iron features.

VII Microquasar Workshop: Microquasars and Beyond

September 1-5 2008

Foca, Izmir, Turkey

\footnotetext{
${ }^{*}$ Speaker.
} 


\section{Overview}

This work is devoted to a discussion of our current understanding of accreting black hole systems when they are in the hard state. In this work, I start by describing the defining properties of the hard state and the questions that we would like to answer concerning the hard state. Then, I discuss the truncated disk picture, which most people consider to be the standard picture for the hard state, and I present some of the key observational evidence in favor of this picture. Although the picture is successful in providing a natural description for many observations, some recent observations appear to be in conflict with the picture, and I describe some of these recent observations. One of the recent observations is presented our recent paper entitled "Broadband X-ray Spectra of GX 339-4 and the Geometry of Accreting Black Holes in the Hard State" by Tomsick et al. (2008) [41]. Finally, I will discuss some alternate pictures for the hard state as well as some on-going work to provide observational tests for theoretical hard state models.

\section{The Hard State}

For most black hole transients, the hard state is seen at the beginnings and at the ends of outbursts. It is often seen at relatively low mass accretion rate, but mass accretion rate is not the only parameter that determines the state of the system because hysteresis can occur where the transition from the hard state at the beginning of an outburst typically occurs at a higher luminosity level that the transition to the hard state at the end of the outburst [16]. In addition, low-luminosity transients (e.g., XTE J1118+480) can remain in the hard state for their entire outbursts, and occasionally, a high-luminosity transient (e.g., V404 Cyg) can remain in the hard state for its entire outburst [39]. There are also several persistent systems (Cyg X-1, GRS 1758-258, 1E 1740.7-2942) spend most of their time in the hard state.

The hard state is defined by three main characteristics. First, the energy spectrum in the hard state is a power-law in the $\sim 5-100 \mathrm{keV}$ bandpass with a photon index in the range $\Gamma=1.4-2.0$. Although the $\left(v F_{v}\right)$ spectrum often peaks near $100 \mathrm{keV}$ and is exponentially cutoff above this energy [15], some observations suggest that the spectrum in this state can extend to higher energies without a cutoff $[1,2]$. The second defining characteristic is a high level of timing noise in the $\mathrm{X}$-ray band. The threshold value for the hard state defined in [27] is an rms noise level of 10\%, but many systems show rms noise levels going up to $20-50 \%$ [33]. The third defining characteristic is the presence of a steady and powerful compact jet [12]. The compact jet is always seen in the radio band, and it has been resolved for GRS 1915+105 [5] and Cyg X-1 [37]. There is strong evidence that the jet emission extends to the infrared and near-IR [4, 28], and it may extend to even higher energies.

One of the main questions about the hard state that we would like to answer concerns the accretion geometry - specifically, what is the inner radius $\left(R_{\text {in }}\right)$ of the optically thick disk. This is important for understanding the relationship between the optically thick disk and the corona (i.e., the site of the hard X-ray production) as well as constraining the disk/jet connection. One can imagine that the geometry of the optically thick disk may be very important for creating the conditions necessary for the production of detectable compact jets. For example, the jet may rely on matter coming from the optically thick disk, suggesting that a small $R_{\text {in }}$ may be required to feed 
the jet. On the other hand, if there is too much soft X-ray emission incident on the jet, then this can cause significant Compton cooling of the jet electrons, and this suggests that if the disk remains relatively hot (and bright) that a relatively large $R_{\text {in }}$ may be required to avoid cooling the jet. Either way, it is clear that one expects that the interaction between the optically thick disk and the jet to be a significant one, making it important to determine the accretion geometry.

\section{The Truncated Disk Picture}

The idea that the hard X-ray emission in the hard state comes from an optically thin region close to the black hole while the optically thick disk recedes was conceived early-on in the study of accretion disks around black holes [35]. Although the Shapiro, Lightman, \& Eardley (1976) model proved to be unstable, this sort of accretion geometry is viable if advection is considered as in Advection-Dominated Accretion Flow (ADAF) models [32]. It was shown that the ADAF model could generally explain the energy spectra seen for the different black hole states by allowing for changes in mass accretion rate and $R_{\text {in }}$ [11]. Importantly, this model envisions that $R_{\text {in }}$ makes its largest changes as it passes through the intermediate state on its way to the hard state. To be viable, this model requires that $R_{\text {in }}$ becomes very large ( $\sim 100-1000$ gravitational radii, $\left.R_{\mathrm{g}}\right)$ when systems are in the hard state and in quiescence. Although the Esin et al. (1997) work does not consider the presence of jets, a recent review article by Narayan \& McClintock (2008) [31] shows the basic ADAF version of the truncated disk picture. (I note that while this is a good representation of the basic picture, one aspect of the picture that does not match the observations is the indication of a compact jet in the intermediate state. In fact, it has been shown that, during outburst decay, the jet does not turn on while the spectrum is evolving in the intermediate state. It only turns on when the source reaches its hardest level [18].)

In the following subsections we list several observations that are well explained within the truncated disk picture.

\subsection{Changes to the Energy Spectrum}

As black hole systems pass from the soft (or "thermal-dominant" or TD) state to the hard state, the primary emission component changes from being a thermal component from the optically thick disk to the hard power-law component discussed above. In almost all systems, when the thermal component is modeled by a disk-blackbody, the inner disk temperature, $k T_{\text {in }}$, drops from values near $1 \mathrm{keV}$ in the TD state to $<0.3 \mathrm{keV}$ in the hard state [19]. After the drop in temperature, the thermal component is often not detected, especially if the X-ray coverage only includes $>3 \mathrm{keV}$ (e.g., the Rossi X-ray Timing Explorer (RXTE)).

In most cases, it is not clear whether the drop in $k T_{\text {in }}$ is due to a change in $R_{\text {in }}$ or a change in mass accretion rate because for most black hole systems, the extinction is too high to obtain a good measurement of the shape of the thermal component since most of the emission is in the soft X-ray and UV bands. However, good measurements were obtained for one system, XTE J1118+480, because it is relatively nearby, $\sim 1.8 \mathrm{kpc}$, and it is at a high Galactic latitude of $b=+62^{\circ}$, so its extinction is very low. At $0.1 \%$ of the Eddington luminosity $\left(L_{\text {Edd }}\right)$, the optical, UV, and X-ray spectrum was measured, and the optical an UV emission was well-described by a disk-blackbody component with $k T_{\text {in }}=0.02 \mathrm{keV}$. Although different studies which modeled this component found 
different values for $R_{\mathrm{in}}$, the values found were between 110 and $700 R_{\mathrm{g}}[10,3,43]$, which are consistent with expectations for the truncated disk model.

A second spectral feature that is consistent with the truncated disk model is related to the hard power-law tail. If this emission is due to inverse Compontonization by a thermal distribution of electrons, then the corona where the hard X-ray emission is produced must be photon-starved in order to maintain a temperature as high as $100 \mathrm{keV}$ [8]. While this is consistent with a truncated disk, and a truncated disk seems to be the most natural explanation, other possibilities include a drop in the radiative efficiency in the disk or other geometrical changes that might somehow isolate the corona from soft X-ray emission produced by the optically thick disk.

\subsection{Drop in Characteristic Frequencies}

The evolution of the timing properties during state transitions also seem to find a natural explanation within the truncated disk picture. Both Quasi-Periodic Oscillations (QPOs) and other characteristic frequencies detected from black hole systems, such as the break frequency in the power spectrum, exhibit a decrease as the mass accretion rate drops during the transition to the hard state and in the hard state. Furthermore, several groups have pointed out that there is a strong correlation between the QPO frequency and the power-law index, $\Gamma[14,17,42,39,36]$, connecting the timing changes to spectral changes that are well-explained by a truncated disk. The example shown in [39] shows that the typical behavior is for the QPO frequency to change from $\sim 10 \mathrm{~Hz}$ to $\sim 0.2 \mathrm{~Hz}$ as $\Gamma$ changes from 2.4 to 1.6 . If this frequency scales with the dynamical time scale at $R_{\text {in }}$, then such a change in frequency would imply a change in $R_{\text {in }}$ by a factor of 14 , so that if $R_{\text {in }}$ was at $6 R_{g}$ in the TD state, then it would be at $84 R_{g}$ by the time the system reached the hard state. Although the QPO is usually not detected in fainter hard states, there is evidence that characteristic frequencies continue to drop in the hard state as flux decreases [40].

\subsection{Drop in the Strength of the Reflection Component}

Another spectral feature that is strongly dependent on the geometry of the system is the reflection component, whose strength depends on the solid angle subtended by the optically thick disk as seen from the hard X-ray source, $\Omega / 2 \pi$. The Compton reflection component consists of a bump in the emission bewtween $\sim 20$ and $100 \mathrm{keV}$ [21] as well as absorption edges and fluorescent emission lines, most prominently from iron.

An important indication that the accretion geometry changes as black hole systems enter the hard state is that $\Omega / 2 \pi$ shows a dramatic drop. It was shown that several black hole systems show very similar changes, specifically, that $\Omega / 2 \pi$ changes from $\sim 1$ to $\sim 0.3$ as $\Gamma$ changes from 2.2 to $1.5[45,44]$. It was also shown that this evolution can be well explained by the truncated disk picture along with a geometry where there is some overlap between the corona and the optically thick disk [44]. This explanation is not necessarily a unique explanation for the evolution, but the good agreement between this model and the data indicate that it is a possible explanation.

\section{Recent Observations that may Conflict with the Truncated Disk Picture}

While the observations described in the preceeding section can be seen as supporting the truncated disk picture, some recent observations present challenges to this picture. In part, improved 
$\mathrm{X}$-ray instrumentation over the past several years has provided this new information, including the better soft X-ray sensitivity and/or better energy resolution of XMM-Newton, the Chandra X-ray Observatory, Swift, and Suzaku when compared with previous satellites. Here, the two challenges we describe and discuss are measurements of the thermal disk component in the hard state and the broad iron features in the reflection component.

\subsection{Thermal Disk Components}

Although it has long been known that sources in relatively bright hard states can exhibit thermal disk components with temperatures of $k T_{\text {in }} \sim 0.2 \mathrm{keV}$, recent observations have shown that this component is present in more sources and at lower luminosities than seen previously. For Cyg X-1, this component has been detected by satellites from ASCA [9] to BeppoSAX [6] to Suzaku [24] when the source has been at a luminosity of $\sim 2 \% L_{\text {Edd }}$. It has also been detected at around this luminosity for GX 339-4 and GRO J1655-40, but there have been reports that it persists at a level of $k T_{\text {in }} \sim 0.2 \mathrm{keV}$ to much lower luminosities, $\sim 0.1 \% L_{\text {Edd }}$, for Swift J1753.5-0127 and XTE J1817-330 [30, 34].

From modeling these components, it has been suggested that they are consistent with little or no change in $R_{\text {in }}$ relative to the location of the inner radius in the TD state [30,34]. The finding in the case of XTE J1817-330 is that the evolution of the properties of the thermal component during $\sim 20$ Swift observations is consistent with an $L \propto T_{\text {in }}^{4}$ relationship, implying that any changes in the thermal disk component is related to a change in mass accretion rate rather than a change in $R_{\text {in }}$. However, obtaining physically meaningful parameters by fitting thermal disk components is challenging, and there are many uncertainties that can introduce systematic errors such as the assumptions about the inner boundary condition, the viscosity prescription, electron scattering that can cause a difference between the effective temperature and the color temperature, and changes to the temperature profile in the disk due to irradiation. In fact, when the same Swift observations that were used to conclude that the inner radius does not change, were re-analyzed accounting for a different inner boundary condition and irradiation of the disk, it was found that the inner radius could change by as much as a factor of 10 [13].

In our recent work on Swift and RXTE observations of GX 339-4 in the hard state, we also detect a thermal disk component at $k T_{\text {in }}=0.193 \pm 0.012 \mathrm{keV}$ at a luminosity of $2.3 \% L_{\text {Edd }}$. The normalization of the disk-blackbody component is related to the inner radius according to $N_{\mathrm{DBB}} \propto$ $R_{\text {in }}^{2}$, and we measure a value of $N_{\text {DBB }}=\left(2.9_{-1.4}^{+2.7}\right) \times 10^{4}$ [41]. GX 339-4 was also observed at a higher luminosity, 5.6\% $L_{\text {Edd }}$, and a higher thermal disk temperature was measured, $k T_{\text {in }}=0.39 \pm$ $0.04 \mathrm{keV}$. However, the value of $N_{\mathrm{DBB}}$ of $700 \pm 200$ is much lower than seen in our observation of GX 339-4 [29], which could be taken as evidence that $R_{\text {in }}$ increases between the two observations. While this could be the case, this conclusion is not consistent with the iron line measurements reported in [41] and [29] and discussed below, and it is not clear how large the systematic error is on the measurement of $R_{\text {in }}$ using the thermal disk component.

Overall, my conclusion concerning using thermal disk components to constrain the evolution of $R_{\text {in }}$ is that it is difficult to be sure that the systematic errors are well-understood. However, it is interesting that sources like Swift J1753.5-0127 and XTE J1817-330 can show disk temperatures of $\sim 0.2 \mathrm{keV}$ while XTE J1118+480 has a disk temperature that is an order of magnitude lower, 
$0.02 \mathrm{keV}$ at the same luminosity level, $\sim 0.1 \% L_{\text {Edd }}$. Even if this does not give a clean measurement of $R_{\text {in }}$, efforts to improve our understanding of this component are still worthwhile.

\subsection{Broad Iron Features in the Hard State}

The second challenge to the truncated disk model is the detection of broad iron features in the hard state. As discussed above, these features are part of the Compton reflection component, and the leading interpretation for the width of the features is that they are relativistically smeared by motion of accretion disk material around the black hole, causing Doppler shifts, as well as by redshifts caused by the black hole's gravitational field. Although such features have been seen in several Galactic black hole systems when they are at high luminosities, the hard state has not been as well-studied with the higher energy resolution available with the current generation of Xray satellites. However, there are now a few hard state iron line studies that use higher resolution instrumentation along with spectral models that include the relativistic effects. The main source that has been studied in this way is GX 339-4, and here we describe results that have been obtained for this source with XMM-Newton, Swift, and RXTE over the past few years. We note that in deriving the Eddington-scaled luminosities, we assumed a distance of $8 \mathrm{kpc}$ for GX 339-4 and a black hole mass of 5.8 solar masses.

Figure 1 shows the RXTE Proportional Counter Array (PCA) light curve and hardness ratio for GX 339-4 from 2004-2008. The system normally has a high level of activity, but the amount of activity over this 4 year period is exceptionally high with 3 separate outbursts. The first study of iron features was carried out using XMM-Newton and RXTE data from 2004. The observation occurred at the start of the 2004-2005 outburst at a relatively high luminosity, 5.6\% $L_{\text {Edd }}$, and the hardness ratio shows that the system was in the hard state. The results of the XMM-Newton plus $R X T E$ spectral analysis are reported in [29]. The spectra show evidence for broad iron features, and they are fitted with relativistic smearing (the "kdblur" model in XSPEC) and two different reflection models ("pexriv" and the "constant density ionized disk" or "CDID" model). The result is that the smearing of the iron features is strong enough to require that the disk extends very close to the black hole. With the first reflection model, $R_{\text {in }}$ is constrained to be $4.0 \pm 0.5 R_{\mathrm{g}}$ while $5.0 \pm 0.5 R_{\mathrm{g}}$ is obtained with the CDID model.

A separate study of GX 339-4 was carried out by our group. We used the Swift and RXTE observations marked in Figure 1, and it is notable that these observations were carried out at the end of the 2007-2008 outburst (in contrast to the XMM-Newton observation described above), and the Swift observations occurred at lower luminosity, $2.3 \%$ and $0.8 \% L_{\text {Edd }}$, than the earlier observation. Even so, the Swift and RXTE spectra also show strong evidence for broad iron features [41]. Our result using the pexriv reflection model smeared by kdblur is that $R_{\text {in }}$ is constrained to be $3.6_{-1.0}^{+1.4}$ $R_{\mathrm{g}}$ at $2.3 \% L_{\mathrm{Edd}}$ and $<10 R_{\mathrm{g}}$ at $0.8 \% L_{\mathrm{Edd}}$. The spectrum at the higher luminosity level is shown in Figure 2.

Although GX 339-4 is the only system with published studies that have used the most recent instrumentation and relativistic reflection models, broad iron features have also been reported for Cyg X-1 [24] and for XTE J1650-500 [7]. Thus, the features are not simply restricted to GX 3394. For GX 339-4, the small inner radii derived are in contradiction to what is expected for the truncated disk model, and this implies that, at least down to $2.3 \% L_{\text {Edd }}$, either the truncated disk picture is wrong or we are mis-interpreting the broad reflection features. 


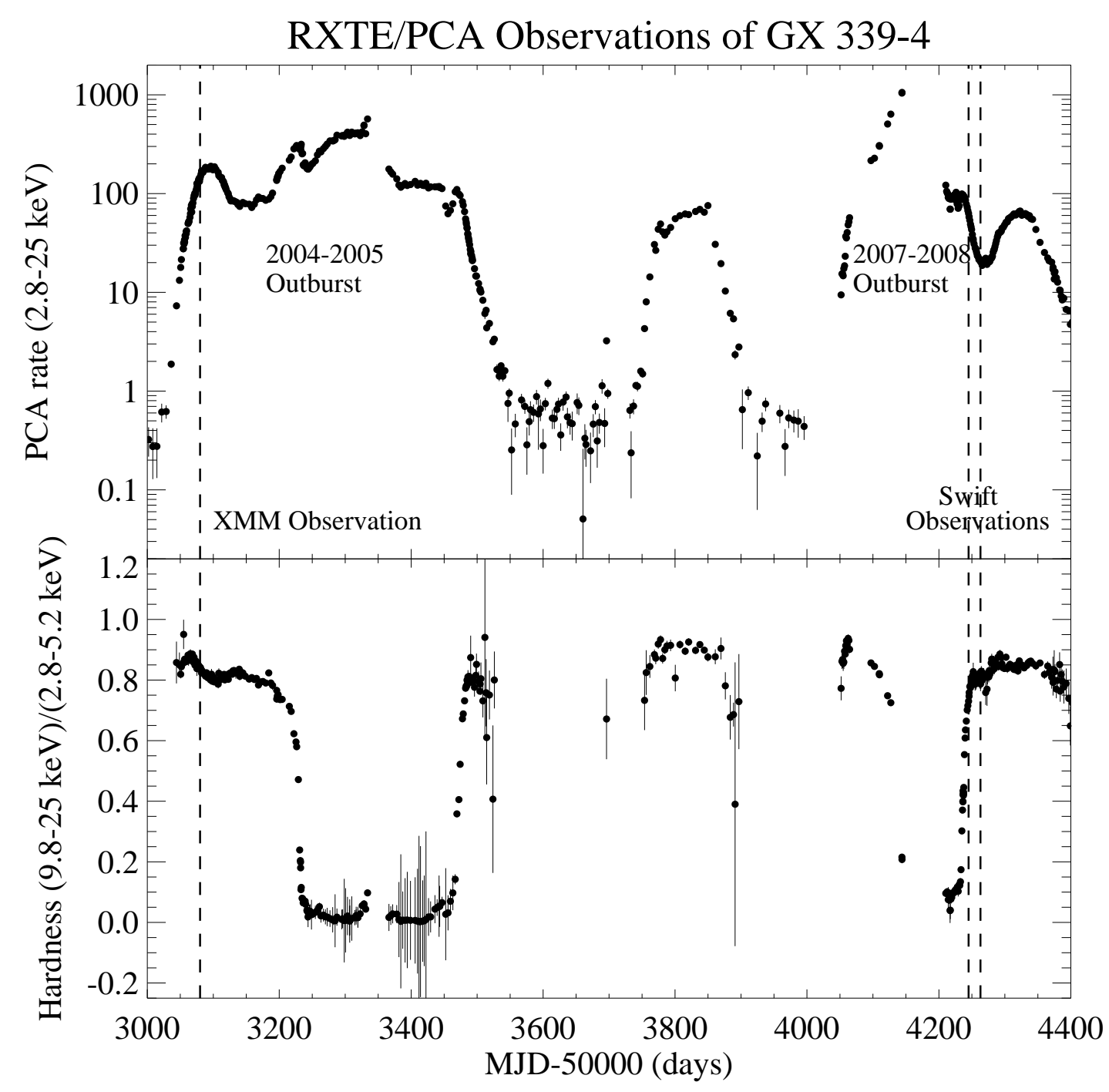

Figure 1: GX 339-4 light curve and hardness ratio from pointed observations with the Rossi X-ray Timing Explorer's Proportional Counter Array (PCA) instrument. Three separate outbursts were detected between 2004 and 2008. The vertical dashed lines mark an XMM-Newton observation in 2004 and two Swift observations in 2007.

\section{Some Alternate Pictures}

Other hard state accretion geometries have been suggested, and it is worthwhile to consider whether they may provide a better explaination for the observations than the truncated disk model. Here, I will consider a compact jet model and a picture where an inner cool disk remains in the hard state.

\subsection{Compact Jet Model}

First, it should be noted that the compact jet model is not necessarily incompatible with the truncated disk picture since it is possible that the compact jet is fed by an ADAF. However, a 


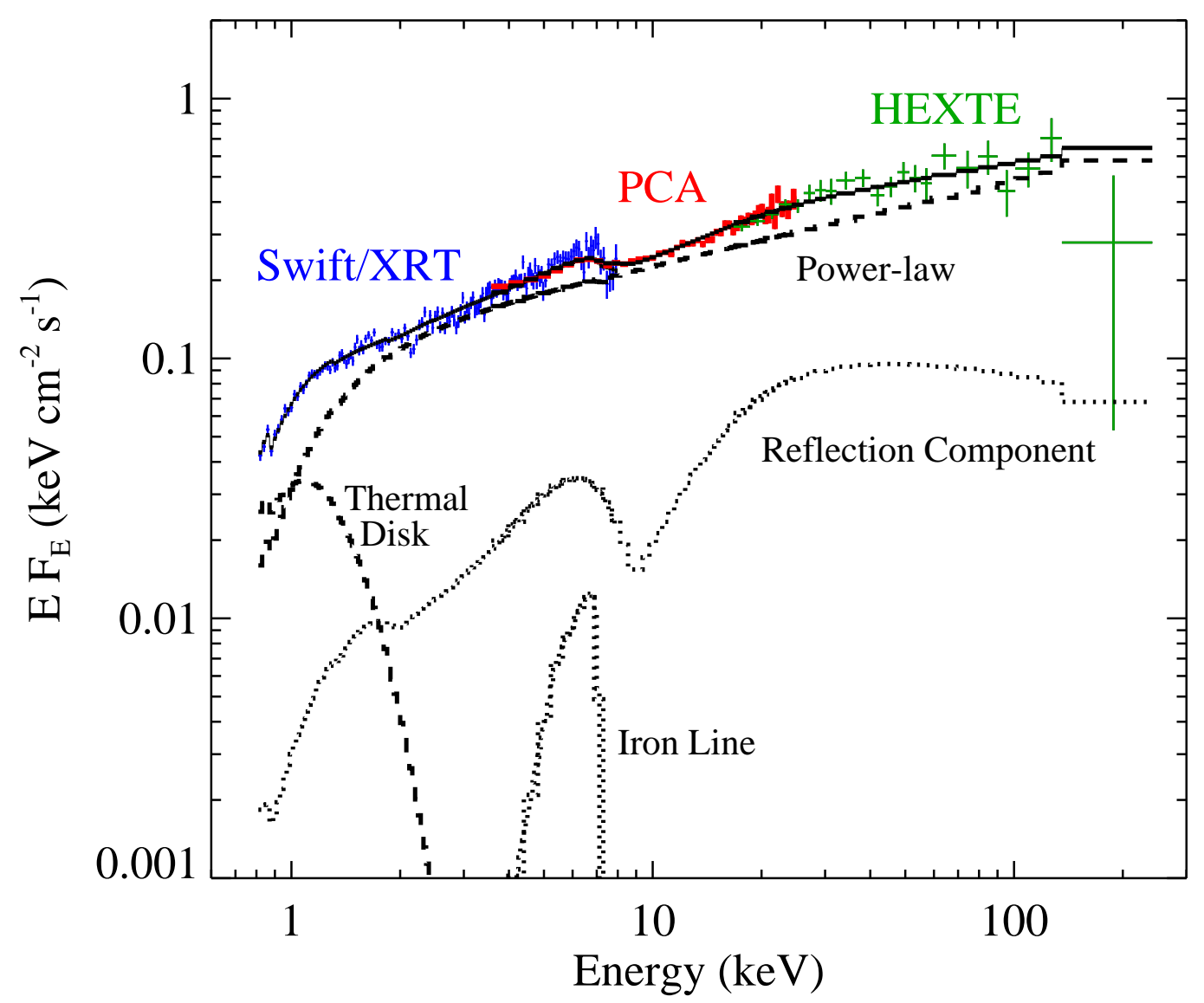

Figure 2: The Swift and RXTE energy spectrum of GX 339-4 taken in 2007 May when the source was in the hard state at a luminosity of $2.3 \% L_{\text {Edd }}$. The model components are shown, and we focus on the thermal disk component and the broad reflection features in this work. The figure has been adapted from Tomsick et al. (2008) [41].

version of the compact jet model that may be able to explain the broad iron features would have the optically thick disk extending within several $R_{g}$ of the black hole while the jet is also launched and collimated in this region. In addition, if a significant fraction of the X-ray emission came from the base of the jet as envisioned by Markoff, Nowak, \& Wilms (2005) [26], then this would give a reflection component from the inner part of the disk that could explain the broad iron features.

In fact, the Markoff et al. [26] compact jet model has now been applied to hard state Spectral Energy Distributions for a few black hole systems. One parameter in the model is the "nozzle radius," which is the radius of the base of the jet. In the spectral model, this parameter is related to the location of the break from optically thick to optically thin synchrotron emission, and it has been relatively well-constrained by some SEDs. When the SEDs for Cyg X-1, GX 339-4, and GRO J1655-40 have been fitted, values for this parameter have ranged from 3.5 to $9.6 R_{g}[26,28]$, which is similar to the values of $R_{\text {in }}$ that are derived from the relativistic reflection models.

However, even if such a picture appears to be consistent with the broadened iron features, it 
is still necessary to ask whether it can explain the overall strength of the reflection component, the shape of the hard X-ray spectrum, and the changes in characteristic frequencies. Although some have argued that such a picture would lead to a much higher value for $\Omega / 2 \pi$, Markoff $\&$ Nowak (2004) [25] have shown that the model is consistent with a drop in this parameter as sources make transitions to the hard state because the X-ray emission in the jet will be relativistically beamed away from the optically thick disk, explaining the drop in $\Omega / 2 \pi$. Concerning the shape of the $\mathrm{X}$-ray spectrum, it is important to determine if black hole spectra always have an cutoff near 100 $\mathrm{keV}$ or not. However, we do know that the spectra sometimes have exponential cutoffs near this energy [15]. The compact jet model does predict cutoffs in the spectra, but it would be useful to determine if the model can specifically predict some of the hard X-ray spectra where the cutoff shape has been very well-measured. Concerning the characteristic frequencies, it is currently an open question whether QPOs would be expected for an X-ray emitting compact jet.

\subsection{Inner Cool Disk Picture}

Another possibility that has been explored in some detail recently is the idea that an inner optically thick disk can remain in the hard state. As the mass accretion rate drops during the transition to the hard state, the truncated disk picture envisions that material from the optically thick disk will evaporate into the corona starting from the inner edge of the disk. However, calculations by [22] that account for viscous generation of heat in the corona and conduction of that heat into the optically thick disk show that the maximum evaporation rate should actually be at $\sim 50$ to a few hundred gravitational radii (depending mostly on viscosity in the corona, i.e., the $\alpha$ parameter). Since the inner disk has the strongest level of soft photon emission, when Compton cooling of the corona is also accounted for, the effect becomes even more pronounced [22]. Thus, not only is it possible for a inner cool disk to remain, but it is actually what calculations predict should happen.

Recently, [23] and [38] have applied the inner cool disk model to observations by determining whether the model can reproduce the thermal disk components seen in GX 339-4 and Swift J1753.5-0127. In fact, it was found that for viscosity parameters in the range $\alpha \sim 0.1-0.4$, the thermal disk components could be reproduced. For the 2007 observations of GX 339-4 with a thermal disk temperature of $T_{\text {eff }}=0.203 \mathrm{keV}$ and a luminosity of $2.3 \% L_{\text {Edd }}$ [41], it was shown that the observations could be reproduced with $\alpha=0.31$, an Eddington-scaled mass accretion rate of $6.87 \%$, and an inner optically thick cool disk extending from the marginally stable orbit (i.e., 1-6 $R_{\mathrm{g}}$, depending on the black hole's spin) to $142 R_{\mathrm{g}}$.

While it has been shown that the model can explain the thermal disk component in the hard state, it has not yet been determined whether it could explain the broadened iron lines. Since the optically thick disk extends to the marginally stable orbit in the model, reflection from that region should certainly produce the broad iron features that are observed. However, it is not clear if the geometry of the hard X-ray source is consistent with most of the hard X-rays illuminating the inner part of the disk. This would be a very useful theoretical study to carry out.

Finally, it is a prediction of the inner cool disk model that when the mass accretion rate becomes low enough the entire inner disk will eventually evaporate. The calculations of [23] and [38] suggests that the complete evaporation of the inner part of the disk should occur at a luminosity near $0.1 \% L_{\text {Edd }}$. 


\section{Summary and Future Work}

The most important point is that it really seems like the observations are telling us that there is a problem either with the truncated disk picture or with our interpretation of the broad iron features for black holes in the hard state. While thus far, the observations only indicate a problem down to a luminosity of $\sim 1 \% L_{\mathrm{Edd}}$, there are strong implications for our entire understanding of the differences between the most fundamental black hole spectral states: Soft (or TD) and hard. Although the thermal disk component may also indicate a problem down to $0.1 \%$, the evidence that this component requires a non-truncated disk is weaker than the broad iron features.

Thus, it is very important to determine if the relativistic interpretation for the broad iron features is the only viable one. It has been suggested that the red wing of the iron line could be explained by Compton down-scattering of the line emission [20]; however, it is still not clear if this model can simultaneously explain the other properties of the hard state. Also, it has been mentioned that it is not clear if the relativistic models are adequate for the case of stellar mass black holes. Although they are often applied to the stellar mass case, the models mentioned above (pexriv and CDID) were originally developed for Active Galactic Nuclei (AGN). Some inaccuracies may be present when using pexriv for hotter accretion disks because of the way it deals with higher ionizations. While the CDID model deals with hot disks (thermal motions and higher ionization states) in a much improved manner, it is still true that the matter densities that are assumed are appropriate for AGN. Developing a relativistic reflection model specifically for stellar mass black holes would be a positive step.

Also, more can be done on the observational side. We currently have programs to try to extend the hard state studies to lower luminosities with XMM-Newton, Suzaku, and Swift. In particular, we recently (2008 September) carried out a 100 ks Suzaku observation of GX 339-4 at a significantly lower luminosity than the [41] Swift and RXTE study. It will be very interesting to see if we find any change in the broad iron features.

Acknowledgements I would especially like to thank Emrah Kalemci for all his efforts in hosting this workshop. I would also like to thank the Local and Scientific Organizing Committees for helping to make the meeting a great success. I acknowledge many contributions from my collaborators, including Stephane Corbel, Emrah Kalemci, Phil Kaaret, Sera Markoff, Simone Migliari, Rob Fender, Charles Bailyn, and Michelle Buxton. I also acknowledge useful discussions with participants of the workshop. I acknowledge partial support from NASA RXTE Guest Observer grants NNG06GA81G and NNX06AG83G and from NASA Swift Guest Observer grant NNX08AQ60G.

\section{References}

[1] Belloni, T., et al., 2006, MNRAS, 367, 1113

[2] Caballero-Garcia, M. D., et al., 2007, ApJ, 669, 534

[3] Chaty, S., Haswell, C. A., Malzac, J., Hynes, R. I., Shrader, C. R., \& Cui, W., 2003, MNRAS, 346, 689

[4] Corbel, S., \& Fender, R. P., 2002, ApJ, 573, L35

[5] Dhawan, V., Mirabel, I. F., \& Rodríguez, L. F., 2000, ApJ, 543, 373 
[6] Di Salvo, T., Done, C., Życki, P. T., Burderi, L., \& Robba, N. R., 2001, ApJ, 547, 1024

[7] Done, C., \& Gierliński, M., 2006, MNRAS, 367, 659

[8] Done, C., Gierliński, M., \& Kubota, A., 2007, A\&A Rev., 15, 1

[9] Ebisawa, K., Ueda, Y., Inoue, H., Tanaka, Y., \& White, N. E., 1996, ApJ, 467, 419

[10] Esin, A. A., McClintock, J. E., Drake, J. J., Garcia, M. R., Haswell, C. A., Hynes, R. I., \& Muno, M. P., 2001, ApJ, 555, 483

[11] Esin, A. A., McClintock, J. E., \& Narayan, R., 1997, ApJ, 489, 865

[12] Fender, R. P., 2001, MNRAS, 322, 31

[13] Gierliński, M., Done, C., \& Page, K., 2008, MNRAS, 388, 753

[14] Gilfanov, M., Churazov, E., \& Revnivtsev, M., 1999, A\&A, 352, 182

[15] Grove, J. E., Johnson, W. N., Kroeger, R. A., McNaron-Brown, K., Skibo, J. G., \& Phlips, B. F., 1998, ApJ, 500, 899

[16] Homan, J., \& Belloni, T., 2005, Ap\&SS, 300, 107

[17] Kalemci, E., 2002, Ph.D. Thesis (UC San Diego), "Temporal Studies of Black Hole X-Ray Transients During Outburst Decay"

[18] Kalemci, E., Tomsick, J. A., Buxton, M. M., Rothschild, R. E., Pottschmidt, K., Corbel, S., Brocksopp, C., \& Kaaret, P., 2005, ApJ, 622, 508

[19] Kalemci, E., Tomsick, J. A., Rothschild, R. E., Pottschmidt, K., \& Kaaret, P., 2004, ApJ, 603, 231

[20] Laurent, P., \& Titarchuk, L., 2007, ApJ, 656, 1056

[21] Lightman, A. P., \& White, T. R., 1988, ApJ, 335, 57

[22] Liu, B. F., Mineshige, S., Meyer, F., Meyer-Hofmeister, E., \& Kawaguchi, T., 2002, ApJ, 575, 117

[23] Liu, B. F., Taam, R. E., Meyer-Hofmeister, E., \& Meyer, F., 2007, ApJ, 671, 695

[24] Makishima, K., et al., 2008, PASJ, 60, 585

[25] Markoff, S., \& Nowak, M. A., 2004, ApJ, 609, 972

[26] Markoff, S., Nowak, M. A., \& Wilms, J., 2005, ApJ, 635, 1203

[27] McClintock, J. E., \& Remillard, R. A., 2006, "Black hole binaries", Compact stellar X-ray sources. Edited by Walter Lewin \& Michiel van der Klis: Cambridge University Press), 157

[28] Migliari, S., et al., 2007, ApJ, 670, 610

[29] Miller, J. M., Homan, J., Steeghs, D., Rupen, M., Hunstead, R. W., Wijnands, R., Charles, P. A., \& Fabian, A. C., 2006a, ApJ, 653, 525

[30] Miller, J. M., Homan, J., \& Miniutti, G., 2006b, ApJ, 652, L113

[31] Narayan, R., \& McClintock, J. E., 2008, New Astronomy Review, 51, 733

[32] Narayan, R., \& Yi, I., 1994, ApJ, 428, L13

[33] Pottschmidt, K., et al., 2003, A\&A, 407, 1039

[34] Rykoff, E. S., Miller, J. M., Steeghs, D., \& Torres, M. A. P., 2007, ApJ, 666, 1129

[35] Shapiro, S. L., Lightman, A. P., \& Eardley, D. M., 1976, ApJ, 204, 187 
[36] Shaposhnikov, N., \& Titarchuk, L., 2007, ApJ, 663, 445

[37] Stirling, A. M., Spencer, R. E., de la Force, C. J., Garrett, M. A., Fender, R. P., \& Ogley, R. N., 2001, MNRAS, 327, 1273

[38] Taam, R. E., Liu, B. F., Meyer, F., \& Meyer-Hofmeister, E., 2008, arXiv:0807.3402 [astro-ph]

[39] Tomsick, J. A., 2004, in Proc. of AIP Conf. Proc. 714: X-Ray Timing 2003: Rossi and Beyond, eds. P. Kaaret, F. K. Lamb, \& J. H. Swank, astro-ph/0401189, 71

[40] Tomsick, J. A., Kalemci, E., \& Kaaret, P., 2004, ApJ, 601, 439

[41] Tomsick, J. A., et al., 2008, ApJ, 680, 593

[42] Vignarca, F., Migliari, S., Belloni, T., Psaltis, D., \& van der Klis, M., 2003, A\&A, 397, 729

[43] Yuan, F., Cui, W., \& Narayan, R., 2005, ApJ, 620, 905

[44] Zdziarski, A. A., Lubiński, P., Gilfanov, M., \& Revnivtsev, M., 2003, MNRAS, 342, 355

[45] Zdziarski, A. A., Lubinski, P., \& Smith, D. A., 1999, MNRAS, 303, L11 